\section{Hábitos alimentarios, obesidad y calidad de vida relacionada con la salud en adolescentes chilenos}

\author{
FABIOLA VILUGRÓN ARAVENA ${ }^{1, a}$, TEMÍSTOCLES MOLINA G., ${ }^{2, b}$, \\ MARÍA EUGENIA GRAS PÉREZ ${ }^{3, c}$, SÍLVIA FONT-MAYOLAS ${ }^{3, \mathrm{~d}}$
}

\section{Association between eating habits and health related quality of life among school age adolescents}

Background: Adolescence is considered a nutritionally vulnerable period of life, since their eating habits are influenced by factors that can promote the consumption of non-nutritious food. Aim: To analyze the relation between eating habits, obesity, and the perception of Health-Related Quality of Life in school-aged adolescents. Material and Methods: A cross-sectional study of 550 adolescents aged $16 \pm 1$ years (60\% women) attending a public education institution in Valparaíso, Chile. The Global School-based Student Health Survey (GSHS) for eating habits and the KIDSCREEN-27 survey for health-related quality of life were self-administered online. The answers were anonymous. A logistic regression analysis was conducted to evaluate the relationship between the variables, which was adjusted for age and sex. Results: Ten percent of men and 6\% of women were obese. Those with an inadequate consumption of fruits had a worst perception of their physical well-being, autonomy and relationship with their parents. Psychological well-being and school environment were perceived worst among those that did not consume breakfast. Obese respondents also reported a poorest physical health. Conclusions: Inadequate eating habits and obesity have a negative effect of health-related quality of life among adolescents.

(Rev Med Chile 2020; 148: 921-929)

Key words: Adolescent; Feeding Behavior; Obesity; Quality of Life.

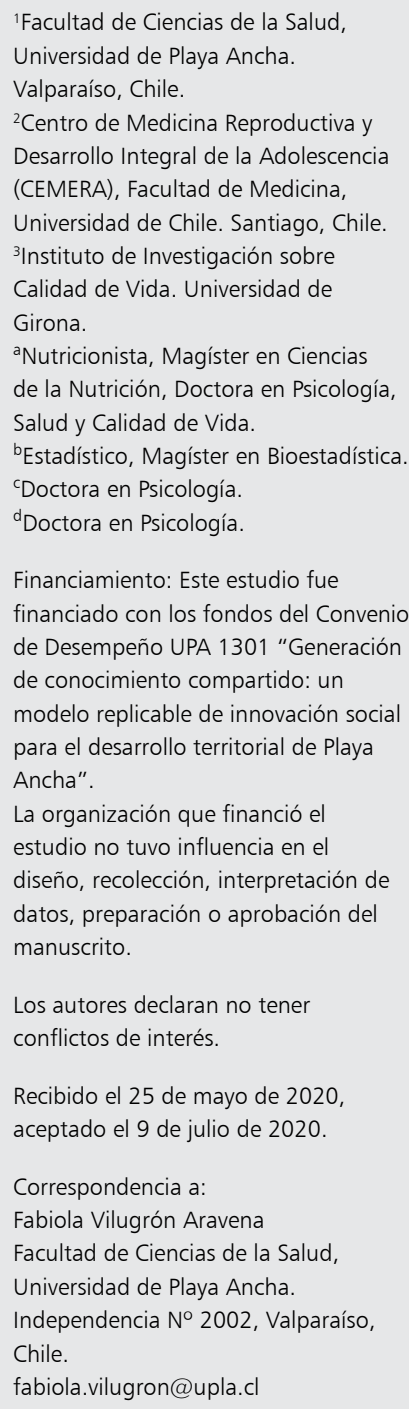

fabiola.vilugron@upla.cl

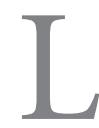

os hábitos alimentarios han sido definidos como "los patrones conductuales regulares qque son observados en algunas especies de interés, y que consta de formas en las cuales sus miembros se procuran el sustento nutritivo, especialmente el tipo de alimento que se ingiere y cómo se ingiere" ${ }^{\prime}$. La adolescencia se considera una etapa nutricionalmente vulnerable debido a que los hábitos alimentarios están fuertemente influenciados por factores socioculturales, emocionales y conductuales, que ocurren en el contexto y que pueden provocar o reforzar el consumo excesivo de alimentos no nutritivos ${ }^{2-4}$. La causa principal de la obesidad es el desequilibrio entre la ingesta alimentaria y la actividad física. Según los datos reportados por la Organización Mundial de la Salud (OMS) ${ }^{5}$, el 2016 más de 340 millones de niños y adolescentes entre 5 y 19 años de edad fueron diagnosticados con sobrepeso $u$ obesidad. El aumento de adiposidad se relaciona con factores de riesgo cardiometabólicos, diabetes y enfermedad cardiovascular ${ }^{6,7}$.

La Asociación Americana del Corazón (AHA) ${ }^{8}$ aconseja una dieta principalmente a base de frutas, 
verduras, granos enteros y productos lácteos descremados, legumbres, pescados y carnes magras, y enfatizan evitar el consumo de azúcar, grasas saturadas y trans, colesterol, sal añadida, e ingesta energética y de micronutrientes adecuada. Asimismo, en Chile, las Guías Alimentarias $(2013)^{9}$ recomiendan la ingesta de tres porciones de lácteos bajos en grasa diarios, que equivalen a 600 $\mathrm{ml}$; cinco porciones diarias de frutas o verduras, lo que equivale a $400 \mathrm{~g}$; dos veces por semana de leguminosas (porotos, garbanzos, lentejas o arvejas); ingesta de dos veces por semana de pescado; y beber de 1,5 litros de agua diaria, equivalente a seis a ocho vasos de agua diarios.

Sin embargo, la adherencia a estas recomendaciones es baja, los datos publicados por la Encuesta Mundial de Salud Escolar $(2013)^{10}$ provenientes de adolescentes chilenos $(n=2.049)$ indican que solo el $42,5 \%$ consume habitualmente dos o más frutas al día, el 24,9\% consume verduras tres o más veces al día, mientras que el 65,3\% ingiere bebidas gaseosas una o más veces en el día y el $5 \%$ consumen tres días o más veces por semana comida rápida. Adicionalmente, reporta que el $57,2 \%$ presenta exceso de peso, de ellos el 14,5\% tiene obesidad, sin diferencias según sexo. Estudios realizados en Estados Unidos, Europa y en América, coinciden con los reportes chilenos, los que han informado que niños y adolescentes no cumplen con las directrices dietéticas ${ }^{11-13}$.

La calidad de vida relacionada con la salud (CVRS) se refiere a la percepción individual y la evaluación subjetiva de su salud y bienestar dentro de contexto cultural $^{14}$. Detecta problemas que afectan a los adolescentes por sobre las medidas convencionales de salud, y permite establecer comparaciones entre subgrupos con determinadas características ${ }^{15,16}$. La evidencia reciente ha sugerido que la obesidad se asocia negativamente con la CVRS de niños y adolescentes, siendo las dimensiones más afectadas el bienestar físico, social, y la autopercepción ${ }^{17-18}$. A pesar de que la alimentación poco saludable es uno de los comportamiento obesogénico principales y que puede influir en la salud física, mental y psicosocial de los adolescentes, pocos estudios han examinado si la adherencia a un patrón dietético específico podría relacionarse positiva o negativa con la $\mathrm{CVRS}^{19-21}$.

Recientemente, en Australia distintos autores han analizado la relación entre los hábitos alimentarios y la CVRS de adolescentes. Bolton y cols. ${ }^{22}$ estudiaron la asociación entre la calidad de la dieta y la CVRS medida con el cuestionario AQoL-6D. Los resultados indican que los adolescentes alimentados con dieta saludable presentan mejor CVRS que aquellos alimentados con dieta poco saludable. Hinchliff y cols. ${ }^{23}$ examinaron la asociación de las dietas de riesgo con estado de ánimo deprimido, conflicto familiar y cercanía emocional entre padres e hijos mediante la aplicación del Short Mood and Feelings y evidenciaron que el conflicto familiar y el estado de ánimo deprimido de los adolescentes se asoció con una dieta riesgosa. Jacka y cols. ${ }^{24}$ analizaron la calidad de la dieta con el estado de ánimo depresivo medido con el cuestionario Short Mood and Feelings, demostrando una asociación positiva entre la dieta saludable y salud mental. Posteriormente, Jacka y cols. ${ }^{25}$ realizaron una investigación similar en el Reino Unido encontrando resultados concordantes con estudios observacionales previos de relaciones entre medidas de calidad de la dieta y problemas de salud mental. Por otra parte, en España, Zaldívar y cols. ${ }^{26}$ relacionaron distintos indicadores de salud física y hábitos de vida saludables con la CVRS medida con el cuestionario KIDSCREEN-10, y evidenciaron una peor percepción de la CVRS en adolescentes con consumo frecuente de comida rápida.

Aunque algunos estudios muestran la asociación entre hábitos alimentarios y CVRS, no existen trabajos que incorporen la obesidad entre sus variables. El objetivo de este estudio es analizar la relación entre los hábitos alimentarios, la obesidad y la CVRS en adolescentes escolarizados de 15 a 18 años matriculados en una escuela secundaria de Valparaíso, Chile.

\section{Material y Método}

Este artículo informa sobre parte de un estudio más amplio que investiga la asociación entre comportamientos de riesgo para la salud y calidad de vida en adolescentes escolarizados.

Esta investigación es de tipo transversal analítico realizada entre mayo y agosto de 2015.

Los adolescentes participantes fueron reclutados de un establecimiento de educación secundaria con dependencia administrativa de la Municipalidad de Valparaíso, seleccionado por conveniencia. Los criterios de inclusión fueron 
tener entre 15 y 18 años cumplidos, y contar con el consentimiento firmado de los padres o tutores previo a la aplicación del cuestionario.

El tamaño muestral fue calculado teniendo en cuenta una población de 1.347 adolescentes, una prevalencia estimada de $p=0,331$, definido a partir de las prevalencias de los comportamientos de riesgo de Chile 9 , un intervalo de confianza de $95 \%$ y un error de muestreo de $4 \%$, obteniéndose un $\mathrm{n}=457$. Para la selección de los participantes se consideró un muestreo por conglomerado considerando cada curso un conglomerado $(\mathrm{n}=38)$, de ellos 18 fueron escogidos utilizando una tabla de números aleatorios. 614 cumplían con los criterios de inclusión, 556 fueron autorizados a participar por medio del consentimiento informado y 6 no consintieron contestar. La muestra para el análisis fue de 550 adolescentes, 59,8\% fueron mujeres. La edad promedio fue $16,07 \pm 0,98$ años (rango 15-18 años).

Los participantes completaron un cuestionario auto aplicado instalado en línea en las aulas de computación del establecimiento educacional en presencia del profesor a cargo, la investigadora responsable expuso el objetivo de la investigación y respondió las consultas. El tiempo promedio de aplicación por curso fue de 35 minutos y los datos obtenidos fueron anónimos.

\section{Variables dependientes}

Calidad de Vida Relacionada con la Salud (CVRS): Se determinó mediante el cuestionario KIDSCREEN 27 en su versión validada para niños y adolescentes chilenos ${ }^{27}$. Está compuesto por una selección de 27 preguntas del KIDSCREEN-52 ${ }^{28}$ agrupados en 5 dimensiones: 1) Bienestar físico; 2) Bienestar psicológico; 3) Autonomía y Relaciones con los padres; 4) Apoyo social y pares; y 5) Entorno escolar. Las respuestas se presentan en una escala de Likert de 5 puntos que evalúa la frecuencia (desde nunca $=1$, hasta siempre $=5$ ) o la intensidad de la respuesta (desde no en absoluto $=1$, hasta muchísimo $=5$ ). Esta versión informó un alfa de Cronbach para el cuestionario completo de 0,890 , similar al resultado obtenido en nuestro estudio $(0,913)$.

\section{Variables independientes}

Hábitos alimentarios: Se utilizaron ocho preguntas extraídas del módulo de conductas alimentarias de la Encuesta Mundial de Salud Escolar
$(2015)^{29}$, clasificadas como consumo adecuado o inadecuado. Cada ítem se categorizó y recodificó considerando los criterios recomendados por CDC $(2014)^{30}$ (Tabla 1).

Obesidad: El peso corporal y la estatura se obtuvieron mediante el auto-reporte ${ }^{31}$. El puntaje Z IMC según edad se calculó utilizando el programa de la OMS Anthro Plus ${ }^{\circledR}$ v.3 de la OMS ${ }^{32}$. Puntajes mayores o iguales a $+2 \mathrm{DE}$ por encima de la mediana establecida fueron clasificados como obesidad $^{33}$.

Variables sociodemográficas: Sexo: se codificó como hombre $=0$, mujer $=1$; y edad: se consultó en años cumplidos.

\section{Análisis estadístico}

Se realizaron análisis descriptivos de todas las variables para caracterizar la muestra. Se usó el test $\chi^{2}$ para probar la diferencia entre las variables de estudio y el sexo. Los puntajes de cada dimensión del Cuestionario KIDSCREEN 27 se calcularon utilizando el análisis Rash, transformados a valores $\mathrm{T}$, y estandarizados a una media de $50 \mathrm{y}$ una desviación estándar de 10 . Se consideró "superior" cuando puntaje fue $>$ p25, e "inferior" cuando el puntaje fue $\leq \mathrm{p} 25^{34}$. Mediante modelos de regresión logística simple y múltiple se calcularon los odds ratios (OR) con sus respectivos intervalos de confianza (IC) del $95 \%{ }^{35}$ para determinar la asociación entre los puntajes de las dimensiones de CVRS con los hábitos alimentarios y la obesidad: Modelo 0: Regresión logística simple y Modelo 1: Regresión logística múltiple ajustadas por edad y sexo. Para evaluar la bondad del ajuste de los modelos se utilizó la prueba de Hosmer y Lemeshow ${ }^{36}$.

El estudio fue aprobado por el Comité de Bioética de la Universidad de Playa Ancha (19 de marzo de 2015) y por el director del establecimiento educacional. Se realizó de acuerdo a los principios éticos de la Declaración de Helsinki. Los adolescentes y sus padres o tutores dieron su asentimiento y consentimiento informado respectivamente antes de participar en la investigación.

\section{Resultados}

La muestra correspondió a 550 adolescentes distribuidos en los cuatro niveles de la enseñanza media de un establecimiento educacional de Valparaíso, como ha sido reportado previamente ${ }^{37}$. 
Tabla 1. Operacionalización de las variables de ingesta alimentaria

\begin{tabular}{|c|c|c|c|}
\hline Variable & Ítem & $\begin{array}{l}\text { Recomendación de } \\
\text { ingesta alimentaria9 }\end{array}$ & Codificación \\
\hline $\begin{array}{l}\text { Consumo de } \\
\text { frutas }\end{array}$ & $\begin{array}{l}\text { Durante los últimos } 30 \text { días, } \\
\text { ¿cuántas veces al día comiste } \\
\text { habitualmente frutas, como } \\
\text { manzana, naranja, plátano, } \\
\text { kiwi, pera u otra? }\end{array}$ & $\begin{array}{c}\text { Consumir al menos } 2 \\
\text { veces al día frutas }\end{array}$ & $\begin{array}{l}\text { Adecuado }=0 \text { - quienes consumieron } 2 \\
\text { o más veces al día durante los últimos } \\
30 \text { días, e inadecuado }=1 \text { - quienes } \\
\text { consumieron menos de } 2 \text { veces al día }\end{array}$ \\
\hline $\begin{array}{l}\text { Consumo de } \\
\text { verduras }\end{array}$ & $\begin{array}{l}\text { Durante los últimos } 30 \text { días, } \\
\text { ¿cuántas veces al día comiste } \\
\text { habitualmente verduras y } \\
\text { hortalizas como lechuga, } \\
\text { repollo, apio, tomate u otra? }\end{array}$ & $\begin{array}{l}\text { Consumir al menos } 3 \\
\text { veces al día verduras }\end{array}$ & $\begin{array}{l}\text { Adecuado }=0 \text { - quienes consumieron } 3 \\
\text { o más veces al día durante los últimos } \\
30 \text { días, e inadecuado }=1 \text { - quienes } \\
\text { consumieron menos de } 3 \text { veces al día }\end{array}$ \\
\hline $\begin{array}{l}\text { Consumo } \\
\text { de leche y } \\
\text { productos } \\
\text { lácteos }\end{array}$ & $\begin{array}{l}\text { Durante los últimos } 30 \text { días, } \\
\text { ¿Cuántas veces por día has } \\
\text { tomado leche o comido algún } \\
\text { producto lácteo como yogurt, } \\
\text { queso, o quesillo? }\end{array}$ & $\begin{array}{l}\text { Consumir al menos } \\
3 \text { veces al día leche y } \\
\text { productos lácteos }\end{array}$ & $\begin{array}{l}\text { Adecuado }=0 \text { - quienes consumieron } 3 \\
\text { o más veces al día durante los últimos } \\
30 \text { días, e inadecuado }=1 \text { - quienes } \\
\text { consumieron menos de } 3 \text { veces al día }\end{array}$ \\
\hline $\begin{array}{l}\text { Consumo de } \\
\text { pescado }\end{array}$ & $\begin{array}{l}\text { ¿Durante los últimos } 30 \\
\text { días, ¿Cuántas veces comiste } \\
\text { pescado? }\end{array}$ & $\begin{array}{l}\text { Consumir al menos } 2 \text { día } \\
\text { a la semana pescado }\end{array}$ & $\begin{array}{l}\text { Adecuado = } 0 \text { - quienes consumieron al } \\
\text { menos } 2 \text { día durante los últimos } 30 \text { días, } \\
\text { e inadecuado }=1 \text { - quienes consumieron } \\
\text { menos de } 2 \text { días durante los últimos }\end{array}$ \\
\hline $\begin{array}{l}\text { Consumo de } \\
\text { agua }\end{array}$ & $\begin{array}{l}\text { Durante los últimos } 30 \text { días } \\
\text { ¿Cuántos vasos de agua } \\
\text { tomaste al día (no considera } \\
\text { bebidas ni jugos)? }\end{array}$ & $\begin{array}{c}\text { Beber } 6 \text { o más vasos de } \\
\text { agua al día }\end{array}$ & $\begin{array}{l}\text { Adecuado }=0 \text { - quienes bebieron } 6 \text { o } \\
\text { más vasos al día durante los últimos } \\
30 \text { días, e inadecuado }=1 \text { - quienes } \\
\text { bebieron menos de } 6 \text { vasos al día }\end{array}$ \\
\hline $\begin{array}{l}\text { Consumo de } \\
\text { desayuno }\end{array}$ & $\begin{array}{l}\text { Durante los últimos } 30 \text { días, } \\
\text { ¿Qué tan frecuente tomaste } \\
\text { desayuno? }\end{array}$ & $\begin{array}{l}\text { Consumir siempre o } \\
\text { habitualmente desayuno }\end{array}$ & $\begin{array}{l}\text { Adecuado }=0 \text { - quienes desayunaron } \\
\text { diariamente durante los últimos } 30 \\
\text { días, e inadecuado }=1 \text { - quienes no } \\
\text { desayunaron diariamente durante los } \\
\text { últimos } 7 \text { días }\end{array}$ \\
\hline $\begin{array}{l}\text { Consumo } \\
\text { de bebidas } \\
\text { gaseosas }\end{array}$ & $\begin{array}{l}\text { Durante los últimos } 30 \text { días, } \\
\text { ¿cuántas veces al día tomaste } \\
\text { gaseosas o bebidas? }\end{array}$ & $\begin{array}{l}\text { Consumir } 2 \text { o menos } \\
\text { veces al día bebidas } \\
\text { gaseosas }\end{array}$ & $\begin{array}{l}\text { Adecuado }=0 \text { - quienes bebieron menos } \\
\text { de una vez al día durante los últimos } \\
30 \text { días, e inadecuado }=1 \text { - quienes } \\
\text { bebieron una o más veces al día }\end{array}$ \\
\hline $\begin{array}{l}\text { Consumo de } \\
\text { comida rápida }\end{array}$ & $\begin{array}{l}\text { Durante los últimos } 7 \text { días, } \\
\text { ¿Cuántos días comiste en un } \\
\text { restaurante de comida rápida? }\end{array}$ & $\begin{array}{l}\text { Consumir } 2 \text { o menos } \\
\text { días a la semana } \\
\text { alimentos en lugares de } \\
\text { comida rápida }\end{array}$ & $\begin{array}{l}\text { Adecuado }=0 \text { - quienes comieron en } \\
\text { lugares de comida rápida } 2 \text { o menos } \\
\text { días en los últimos } 7 \text { días, e inadecuado } \\
=1 \text { - quienes comieron en lugares de } \\
\text { comida rápida al menos } 3 \text { días en los } \\
\text { últimos } 7 \text { días }\end{array}$ \\
\hline
\end{tabular}

El 10,3\% de hombres y el 6,1\% de las mujeres presentó obesidad, sin diferencias significativas según sexo. El alimento con mayor porcentaje de ingesta adecuada fue el consumo de comida rápida $(88,7 \%)$, seguido por el consumo de frutas $(64,5 \%)$, y el consumo de bebidas gaseosas $(47,1 \%)$, sin diferencias según sexo. Menos del $30 \%$ reportó cumplir con las recomendaciones de lácteos, verduras y agua siendo significativamente mayor la ingesta adecuada de lácteos $(32,6 \%)$ y agua $(28,9 \%)$ en los hombres. Solo el $9,3 \%$ informó consumir pescado dos o más veces a la semana y el $42,2 \%$ desayunar diariamente, estos consumos fueron significativamente mayor en los hombres (Tabla 2).

En el análisis inicial del modelo de regresión 
Tabla 2. Porcentaje de adherencia a las recomendaciones de ingesta alimentaria de adolescentes según sexo $(n=550)$

\begin{tabular}{|c|c|c|c|c|c|c|c|}
\hline Variable & $\begin{array}{c}\text { Total } \\
(n=550)\end{array}$ & $\begin{array}{c}\text { Adecuado } \\
\%\end{array}$ & $\begin{array}{l}\text { Hombres } \\
(n=221)\end{array}$ & $\begin{array}{c}\text { Adecuado } \\
\%\end{array}$ & $\begin{array}{l}\text { Mujeres } \\
(n=329)\end{array}$ & $\begin{array}{c}\text { Adecuado } \\
\%\end{array}$ & Valor-p \\
\hline Consumo de frutas & 355 & 64,5 & 142 & 64,2 & 213 & 64,7 & 0,907 \\
\hline Consumo de verduras & 110 & 20,0 & 42 & 19,0 & 68 & 20,7 & 0,632 \\
\hline $\begin{array}{l}\text { Consumo de leche y productos } \\
\text { lácteos }\end{array}$ & 139 & 25,3 & 72 & 32,6 & 67 & 20,4 & 0,001 \\
\hline Consumo de pescado & 51 & 9,3 & 29 & 13,1 & 22 & 6,7 & 0,011 \\
\hline Consumo de agua & 132 & 24,0 & 64 & 28,9 & 68 & 20,7 & 0,026 \\
\hline Consumo de desayuno & 232 & 42,2 & 111 & 50,2 & 121 & 36,8 & 0,002 \\
\hline Consumo de bebidas gaseosas & 259 & 47,1 & 102 & 46,2 & 157 & 47,7 & 0,718 \\
\hline Consumo de comida rápida & 488 & 88,7 & 202 & 91,4 & 286 & 86,9 & 0,104 \\
\hline
\end{tabular}

logística simple (Modelo 0), se observa que la ingesta inadecuada de varios alimentos se asocia con una peor percepción de las dimensiones de la CVRS (Tabla 3).

El análisis de regresión logística múltiple ajustado por edad y sexo (Modelo 1) muestra que para el consumo de frutas, la razón entre adolescentes que tuvieron una percepción mala de la dimensión bienestar físico versus aquellos que tuvieron una percepción buena, es 2,98 veces mayor en los adolescentes con consumo inadecuado en comparación con los adolescentes con consumo adecuado (OR: 2,98; 95\% IC: 1,84-4,83) y la razón entre adolescentes que tuvieron una percepción mala de la dimensión autonomía y relación con los padres versus aquellos que tuvieron una percepción buena, es 1,67 veces mayor en los adolescentes con consumo inadecuado en comparación con los adolescentes con consumo adecuado (OR: 1,67; 95\% IC: $1,03-2,68)$.

Para el consumo lácteos y de productos lácteos, la razón entre adolescentes que tuvieron una percepción mala de la dimensión autonomía y relación con los padres versus aquellos que tuvieron una percepción buena, es 1,81 veces mayor en los adolescentes con consumo inadecuado en comparación con los adolescentes con consumo adecuado (OR: 1,81; 95\% IC: 1,03-3,16).

Para el consumo de desayuno, la razón entre adolescentes que tuvieron una percepción mala de la dimensión bienestar psicológico versus aquellos que tuvieron una percepción buena, es 1,82 veces mayor en los adolescentes con consumo inade- cuado en comparación con los adolescentes con consumo adecuado (OR: 1,82; 95\% IC: 1,18-2,81) y la razón entre adolescentes que tuvieron una percepción mala de la dimensión entorno escolar versus aquellos que tuvieron una percepción buena, es 2,06 veces mayor en los adolescentes con consumo inadecuado en comparación con los adolescentes con consumo adecuado (OR: 2,06; 95\% IC: 1,35-3,12).

Para el peso corporal, la razón entre adolescentes que tuvieron una percepción mala de la dimensión bienestar físico versus aquellos que tuvieron una percepción buena, es 2,25 veces mayor en los adolescentes con obesidad en comparación con los adolescentes sin obesidad (OR: 2,25; 95\% IC: 1,17-4,30) (Tabla 3).

\section{Discusión}

El presente trabajo muestra una gran proporción de adolescentes con baja adherencia a las directrices dietéticas que promueven las Guías Alimentarias de Chile 9 . En comparación con las cifras nacionales ${ }^{10}$, el porcentaje que cumple con las recomendaciones de consumo de comida rápida y de frutas fue mayor, mientras que la prevalencia de obesidad fue ligeramente menor. Si bien son múltiples los factores de riesgo asociados al aumento en la prevalencia de obesidad ${ }^{6}$, estas diferencias pueden ser atribuidas a entornos escolares que promueven y facilitan elecciones saludables, y que involucran la escuela, la familia y la comunidad ${ }^{4}$. 


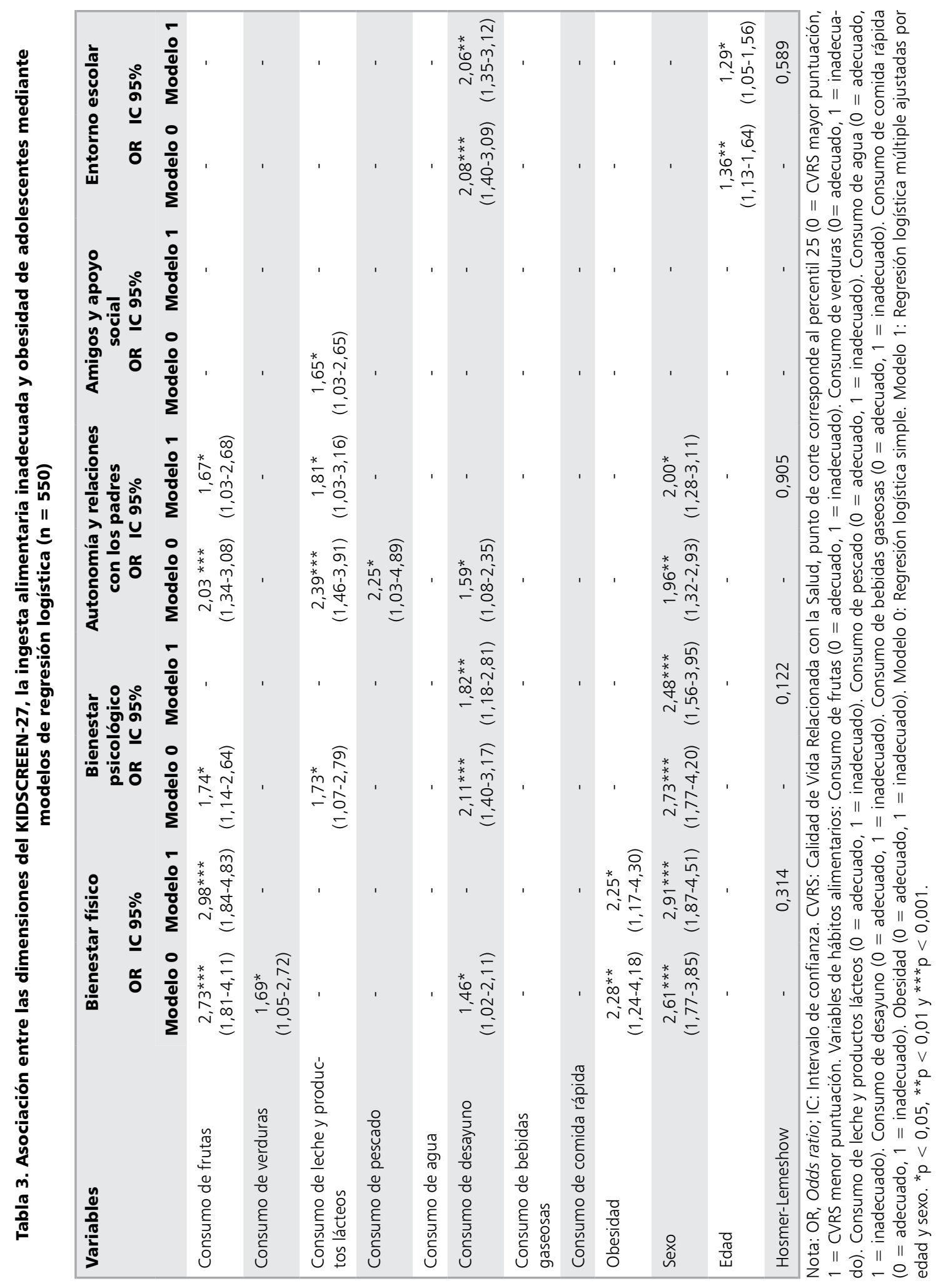


Nuestros resultados evidenciaron que los adolescentes con obesidad y con hábitos alimentarios inadecuados tienen una CVRS significativamente menor que sus pares no obesos y con hábitos alimentarios saludables. Se observó que los adolescentes con obesidad y con consumo inadecuado de frutas reportaron una peor percepción de la dimensión bienestar físico. Ottava y cols. ${ }^{17}$ llegó a similares resultados y encontraron mayores diferencias en las puntuaciones de bienestar físico y de autopercepción. Por el contrario, Cárdenas y cols. ${ }^{18}$ informaron que la CVRS era independiente del peso corporal y reporta que la dimensión más deteriorada por la obesidad es el funcionamiento social, esto sugiere que el problema de obesidad precisa de diferentes miradas, y es percibida por los propios afectados de acuerdo al grupo etario al que pertenezcan y a su contexto socioeconómico y cultural $^{38}$. Es posible que la asociación antes mencionada entre el consumo de frutas y el bienestar físico se atribuya a que una dieta rica en alimentos vegetales e integrales pueden ayudar a reducir el riesgo de obesidad ${ }^{39}$. En particular, Zaldívar y cols. ${ }^{26}$ evidenciaron el consumo frecuente de comida rápida se asociaba con una peor percepción de la CVRS en adolescentes, hallazgo no evidenciado en este estudio.

Además, se identificó que la percepción de las dimensiones de bienestar psicológico y de entorno escolar es peor en los adolescentes que no desayunan habitualmente. Si bien no hay estudios comparables, estos resultados concuerdan parcialmente con Bolton y cols. ${ }^{22}$ quienes informaron que un aumento en la puntuación de la dieta saludable (bajo consumo de bebidas, bajo consumo de comida rápida y consumo habitual de desayuno) se asoció con un aumento en la CVRS de adolescentes urbanos y rurales. En particular, Jacka y cols. ${ }^{24,25}$ encontraron una asociación entre una dieta poco saludable y problemas de salud mental.

Este estudio evidenció que el consumo inadecuado de frutas y de productos lácteos se asoció con una peor percepción de la dimensión de autonomía y relación con los padres. Si bien Hinchliff y cols. ${ }^{23}$ no analizaron la relación entre el consumo de alimentos y la CVRS, reportaron que el conflicto familiar y el estado de ánimo deprimido de los adolescentes se asoció con una dieta no saludable.

Una de las limitaciones del estudio es la homogeneidad de la muestra debido a que estuvo conformada solo por adolescentes matriculados en un establecimiento educacional de Valparaíso. Además, al tratarse de un estudio transversal, no permite inferir causalidad entre las variables.

Agradecimientos: Los autores agradecen al equipo psicosocial, estudiantes y profesores por su cooperación en este estudio.

\section{Referencias}

1. López-Espinoza A, Magaña González C. Hábitos alimentarios: psicobiología y socioantropología de la alimentación, Mc Graw-Hill. México: 2014.

2. World Health Organization. Nutrition in adolescence: issues and challenges for the health sector: issues in adolescent health and development. Geneva: WHO; 2005.

3. Jaworska N, MacQueen G. Adolescence as a unique developmental period. J Psychiatry Neurosci 2015; 40 (5): 291-3. doi: 10.1503/jpn.150268.

4. Wafa SW, Ghazalli R. Association between the school environment and children's body mass index in Terengganu: A cross sectional study. PLoS One 2020; 24; 15 (4): e0232000. doi: 10.1371/journal.pone.0232000. eCollection 2020.

5. Organización Mundial de la Salud. Obesidad y sobrepeso; 2017. Disponible en: https://www.who.int/es/ news-room/fact-sheets/detail/obesity-and-overweight. [Consultado el 22 de abril de 2020].

6. Lee EY, Yoon KH. Epidemic obesity in children and adolescents: risk factors and prevention. Front Med. 2018; 12 (6): 658-66. doi: 10.1007/s11684-018-0640-1.

7. Magge SN, Goodman E, Armstrong SC; Committee on Nutrition; Section On Endocrinology; Section on Obesity. The Metabolic Syndrome in Children and Adolescents: Shifting the Focus to Cardiometabolic Risk Factor Clustering. Pediatrics. 2017; 140 (2). pii: e20171603. doi: 10.1542/peds.2017-1603.

8. Gidding S, Dennison B, Birch L, Daniels S, Gillman M, Lichtenstein A, et al. Dietary Recommendations for Children and Adolescents: A Guide for Practitioners. Pediatrics 2006; 117 (2): 544-59. doi: 10.1542/ peds.2005-2374.

9. Ministerio de Salud de Chile. Estudio para la revisión y actualización de las Guías Alimentarias para la población chilena. Santiago: 2013. Disponible en: http://web.minsal.cl/portal/url/item/dde0bc471a56a001e040010165012224.pdf. [Consultado el 10 de agosto de 2019].

10. Ministerio de Salud de Chile, Organización Mundial de la Salud, Organización Panamericana de la Salud y 
Centro para el Control y la Prevención de Enfermedades. Informe de la Encuesta Mundial de Salud Escolar Chile 2013. Santiago: 2013. Disponible en: http://www. repositoriodigital.minsal.cl/bitstream/handle/2015/518/ Encuesta-de-salud-escolar-2013.pdf?sequence $=1$ \&isAllowed $=y$. [Consultado el 15 de octubre de 2019].

11. Banfield EC, Liu Y, Davis JS, Chang S, Frazier-Wood AC. Poor Adherence to US Dietary Guidelines for Children and Adolescents in the National Health and Nutrition Examination Survey Population. J Acad Nutr Diet. 2016; 116 (1): 21-7. doi: 10.1016/j. jand.2015.08.010.

12. Lynch C, Kristjansdottir AG, Te Velde SJ, Lien N, Roos E, Thorsdottir I, et al. Fruit and vegetable consumption in a sample of 11-year-old children in ten European countries-the PRO GREENS cross-sectional survey. Public Health Nutr 2014; 17 (11): 2436-44. doi: 10.1017/ S1368980014001347.

13. Moreno LA, Gottrand F, Huybrechts I, Ruiz J, González-Gross, M, DeHenauw S. Nutrition and lifestyle in european adolescents: The HELENA (Healthy Lifestyle in Europe by Nutrition in Adolescence) study. Adv Nutr 2014; 5 (5): 615S-23S. doi:10.3945/an.113.005678.

14. Baumann C, Erpelding ML, Perret-Guillaume C, Gautier A, Régat S, Collin JF, et al. Health-related quality of life in French adolescents and adults: norms for the DUKE Health Profile. BMC Public Health 2011; 11: 401. doi: 10.1186/1471-2458-11-401.

15. Simon A, Chan K, Forrest C. Assessment of children's health-Related Quality of Life in the United States with a multidimensional index. Pediatrics 2008; 121: e118-26. doi: 10.1542/peds.2007-0480.

16. Rajmil L, Herdman M. Avances y retos en la medida de la calidad de vida relacionada con la salud en la población infantil y adolescente. An Pediatr (Barc) 2019; 90 (5): 261-2. doi: 10.3389/fphar.2015.00101.

17. Ottova V, Erhart M, Rajmil L, Dettenborn-Betz L, Ravens-Sieberer U. Overweight and its impact on the health-related quality of life in children and adolescents: results from the European KIDSCREEN survey. Qual Life Res 2012; 21: 59-69. doi: 10.1007/s11136-011-99227.

18. Cárdenas VM, Dávila S, Gallegos E, Salazar B, Rizo M. Obesidad y Calidad de Vida Relacionada con la Salud en Adolescentes Escolares. Desarrollo Cientif Enferm 2009; 17 (7): 297-301.

19. Barr JT, Schumacher GE. The need for a nutrition-related quality-of-life measure. J Am Diet Assoc 2003; 103: 177-80. doi: 10.1053/jada.2003.50058.

20. Ruano-Rodríguez C, Serra-Majem L, Dubois D. Assessing the impact of dietary habits on health-related quality of life requires contextual measurement tools. Frontier Pharmacology 2015; 6: 101. doi: 10.3389/ fphar.2015.00101.

21. O'Neil A, Quirk SE, Housden S, Brennan SL, Williams LJ, Pasco JA, et al. Relationship between diet and mental health in children and adolescents: a systematic review. Am J Public Health 2014; 104 (10): e31-42. doi: 10.2105/ AJPH.2014.302110.

22. Bolton K, Jacka F, Allender S, Kremer P, Gibbs L, Waters $\mathrm{E}$, et al. The association between self-reported diet quality and health-related quality of life in rural and urban Australian adolescents. Aust J Rural Health 2016; 24 (5): 317-25. doi: 10.1111/ajr.12275.

23. Hinchliff G, Kelly A, Chan G, Patton G, Williams J. Risky dieting amongst adolescent girls: Associations with family relationship problems and depressed mood. Eat Behav 2016; 22: 222-4. doi: 10.1016/j.eatbeh.2016.06.001.

24. Jacka FN, Kremer PJ, Leslie ER, Berk M, Patton GC, Toumbourou JW, et al. Associations between diet quality and depressed mood in adolescents: results from the Australian Healthy Neighbourhoods Study. Aust NZJ Psychiatry 2010; 44 (5): 435-42. doi: 10.3109/00048670903571598.

25. Jacka FN, Rothon C, Taylor S, Berk M, Stansfeld S. Diet quality and mental health problems in adolescents from East London: a prospective study. Soc Psychiatry Psychiatr Epidemiol 2013; 48 (8): 1297-306. doi: 10.1007/ s00127-012-0623-5.

26. Zaldívar N, Pérez de Albéniz A, Fonseca E. Salud Mental en niños y adolescentes de la Rioja: Resultados de la Encuesta Nacional de Salud de España 2011/12. Contextos Educ 2018; 22: 129-48. doi: 10.18172/con.3410.

27. Molina T, Montaño R, González E, Sepúlveda R, Hidalgo-Rasmussen C, Martínez V, et al. Propiedades psicométricas del cuestionario de calidad de vida relacionada con la salud KIDSCREEN-27 en adolescentes chilenos. Rev Med Chile 2014; 142 (11): 1415-21. doi: 10.4067/ S0034-98872014001100008.

28. Ravens-Sieberer U, Gosch A, Rajmil L, Erhart M, Bruil J, Duer W. KIDSCREEN Group E. KIDSCREEN-52 quality-of-life measure for children and adolescents. Expert Rev of Pharmacoecon Outcomes Res 2005; 5 (3): 353-64. doi: 10.1586/14737167.5.3.353.

29. CDC. Global School-based Student Health Survey (GSHS), Santiago: 2015. Disponible en: http://www. cdc.gov/gshs/index.htm. [Consultado el 1 de enero de 2016].

30. CDC. Encuesta Mundial de Salud escolar Argentina 2014. Disponible en: http://www.msal.gob.ar/ent/images/stories/vigilancia/pdf/2014-09_informe-EMSE-2012. 
pdf. Buenos aires: 2014. [Consultado el 10 de noviembre de 2019].

31. Mei Z, Grummer-Strawn L, Pietrobelli A, Goulding A, Goran M, Dietz W. Validity of body mass index compared with other body-composition screening indexes for the assessment of body fatness in children and adolescents. Am J Clin Nutr 2002; 75 (6): 978-85. doi: 10.1093/ ajcn/75.6.978.

32. Organización Mundial de la Salud (2009). Software Anthro Plus para evaluar el crecimiento y desarrollo de los niños del mundo (versión 3). Disponible en: https:// www.who.int/childgrowth/software/es/. [Consultado el 14 de agosto de 2019].

33. World Health Organization. Growth reference data for 5-19 years [Internet]. WHO. 2007. Disponible en: http://www.who.int/growthref/en/. [Consultado el 14 de agosto de 2019].

34. Rajmil L, Alonso J, Berra S, Ravens-Sieberer U, Gosch A, Simeoni M y KIDSCREEN Group. Use of a children questionnaire of health-related quality of life (KIDSCREEN) as a measure of needs for health care services. J Adolesc Health. 2006; 38 (5): 511-8. doi: 10.1016/j. jadohealth.2005.05.022.

35. Cerda J, Vera C, Rada G. Odds ratio: aspectos teóricos y prácticos. Rev Med Chile 2013; 141 (10): 1329-35. doi: 10.4067/S0034-98872013001000014.

36. Hosmer D, Lemeshow S. Applied Logistic Regression. 2nd ed. Sons JW\&, editor. New York: INC; 2000.

37. Vilugrón F, Hidalgo-Rasmussen C, Molina T, Gras $\mathrm{M}$, Font-Mayolas S. Uso de sustancias psicoactivas y calidad de vida relacionada con la salud en adolescentes escolarizados. Rev Med Chile. 2017; 145: 1525-34. doi: 10.4067/s0034-98872017001201525.

38. Medina FX, Aguilar, Solé-Sedeño JM. Aspectos sociales y culturales sobre la obesidad: reflexiones necesarias desde la salud pública. Nutr. clín. diet. hosp. 2014; 34 (1): 67-71. doi: 10.12873/341.

39. Wall C, Stewart A, Hancox R, Murphy R, Braithwaite I, Beasley R, et al, The ISAAC Phase Three Study Group. Association between Frequency of Consumption of Fruit, Vegetables, Nuts and Pulses and BMI: Analyses of the International Study of Asthma and Allergies in Childhood (ISAAC). Nutrients 2018; 10, 316; doi: 10.3390/nu10030316. 